\title{
Resistencia de Cepas de Staphylococcus aureus Aisladas de la Mucosa Oral de una Población Joven Peruana
}

\author{
Resistance of Staphylococcus aureus Strains Isolated from \\ the Oral Mucosa in a Young Peruvian Population
}

\begin{abstract}
Alfredo Márquez-Oviedo; Ada Carolina Romero-Coasaca; Margarita F. Requena-Mendizábal; Elba MartínezCadillo; Ursula Mongrut-Villegas; Esperanza R. Ayón-Haro \& Augusto S. Guardia-Huamaní
\end{abstract}

MÁRQUEZ-OVIEDO, A.; ROMERO-COASACA, A. C.; REQUENA-MENDIZÁBAL, M. F.; MARTÍNEZ-CADILLO, E.; MONGRUT-VILLEGAS, U.; AYÓN-HARO, E. R. \& GUARDIA-HUAMANÍ, A. S. Resistencia de cepas de Staphylococcus aureus aisladas de la mucosa oral de una población joven peruana. Int. J. Odontostomat., 15(3):634-638, 2021.

RESUMEN: Staphylococcus aureus es un patógeno importante responsable de una variedad de enfermedades infecciosas. La emergencia de cepas resistentes a la meticilina es un tema preocupante. Existen escasos estudios que hayan reportado la resistencia de cepas aisladas de la cavidad bucal. Determinar la sensibilidad antimicrobiana de cepas de Staphylococcus aureus aisladas de mucosa bucal de portadores asintomáticos jóvenes peruanos. Se incluyeron 64 individuos a quienes se tomaron muestras de mucosa oral, mucosa nasal y de manos. Las muestras fueron cultivadas en agar manitol salado. La prueba coagulasa fue empleada para confirmar la presencia de Staphylococcus aureus. Luego se realizó el antibiograma usando el método de Kirby-Bauer. La prevalencia de colonias positivas de S. aureus provenientes de la mucosa oral, mucosa nasal y de la palma de la mano fue 7,8 \%, 15,6 \% y0,0 \%, respectivamente. En todas las muestras, se observaron una prevalencia de sensibilidad menor de $40 \%$ y $11 \%$ a la eritromicina y penicilina, respectivamente. Las colonias de la mucosa oral mostraron resistencia a la penicilina. En todas las muestras, se observó una prevalencia de sensibilidad $\geq 60 \%$ en amoxicilina/ácido clavulánico, co-trimoxazol, gentamicina, oxacilina, ciprofloxacino, vancomicina y ampicilina/sulbactam. Se encontró resistencia a vancomicina en un $10-20 \%$ de todas las muestras. Se encontró una alta prevalencia en la sensibilidad a amoxicilina/ácido clavulánico, gentamicina, co-trimoxazol, oxacilina, ciprofloxacino y ampicilina/sulbactam. Se encontró un elevado porcentaje de resistencia a la vancomicina en muestras de mucosa oral en comparación a otros estudios.

PALABRAS CLAVE: Staphylococcus aureus, pruebas de sensibilidad microbiana, adulto joven.

\section{INTRODUCCIÓN}

Staphylococcus aureus es una bacteria con forma de coco Gram positivo, agrupada generalmente en racimos aerobios y anaerobios facultativos. Staphylococcus aureus es la especie del género Staphylococcus más relacionada con la patología infecciosa humana. Se sabe que esta bacteria ocasiona infecciones en piel, ojos, tracto gastrointestinal, vagina y bacteriemia a partir de focos primarios de heridas traumáticas u operatorias, quemaduras u otras lesiones cutáneas graves (Loomba et al., 2010).
La emergencia de cepas resistentes a la meticilina y a la vancomicina; y además la síntesis masiva de agentes antibacterianos son problemas graves. Esto es debido a la alta mortalidad por las infecciones sistémicas ocasionadas por Staphylococcus aureus meticilino resistente (SAMR) en el ambiente hospitalario (Loomba et al.). Recientemente se ha informado una prevalencia entre 50 a $90 \%$ de cepas SAMR en hospitales del Perú (Mendoza et al., 2003). Staphylococcus aureus se multiplica principalmente en

Laboratorio de Microbiología de la Facultad de Odontología de la Universidad de San Martín de Porres, Lima, Perú.

Fuente de Financiamiento. Facultad de Odontología de la Universidad de San Martín de Porres, Perú.

Received: 2020-04-02 Accepted: 2021-04-2021

634 
las narinas anteriores. Además, esta bacteria se ha podido detectar particularmente en los dedos y palmas de las manos (Zelaya-Trebejo et al., 2001).

Es de suma importancia para la salud pública, tener conocimiento de la diseminación e identificación de las transmisiones o brotes epidémicos. Es necesario que no solo los epidemiólogos y los microbiólogos sino también los odontólogos contribuyan al control y seguimiento de cepas resistentes. De allí que el objetivo de este trabajo fue determinar la sensibilidad antimicrobiana de Staphylococcus aureus en muestras de mucosa bucal de portadores asintomáticos jóvenes en una población peruana.

\section{MATERIAL Y MÉTODO}

Muestra. En este estudio se incluyeron 64 estudiantes registrados en la Asignatura de Microbiología de la Facultad de Odontología de la Universidad de San Martín de Porres de los meses julio-noviembre del año 2011 , seleccionados a través de un muestreo aleatorio. Se obtuvo el consentimiento informado de cada participante de este estudio. El protocolo estuvo de acuerdo con las normas éticas de la Declaración de Helsinki de 1983. Además, éste fue revisado y aprobado por el Consejo Revisor de la Facultad de Odontología de la Universidad de San Martín de Porres. La edad de los participantes estuvo comprendida entre 16 a 22 años. No se consideraron portadores de enfermedad sistémica o aquellos que recibieron antibióticos dentro de los tres meses previos al estudio.

Toma de la muestra. Los hisopados fueron tomados de la mucosa del carrillo. Las muestras nasales fueron recogidas a través de movimientos circulares dentro de un $1 \mathrm{~cm}$ distal de las narinas anteriores. Asimismo, se tomaron las muestras de las palmas de las manos.

Procesamiento de la muestra. Se tomó en cuenta una muestra por paciente. Se realizó la siembra por estrías con el hisopo en placas de agar manitol salado y se incubó a $37^{\circ} \mathrm{C}$ por 24 horas. Se consideró un cultivo manitol positivo a aquellos en los cuales se pudieron observar más de una colonia de color blanco o dorado sugerente de Staphylococcus aureus, en algún cuadrante de la placa de cultivo (Fig. 1). Luego se llevó a cabo la tinción Gram para observar las características microscópicas de Staphylococcus aureus.

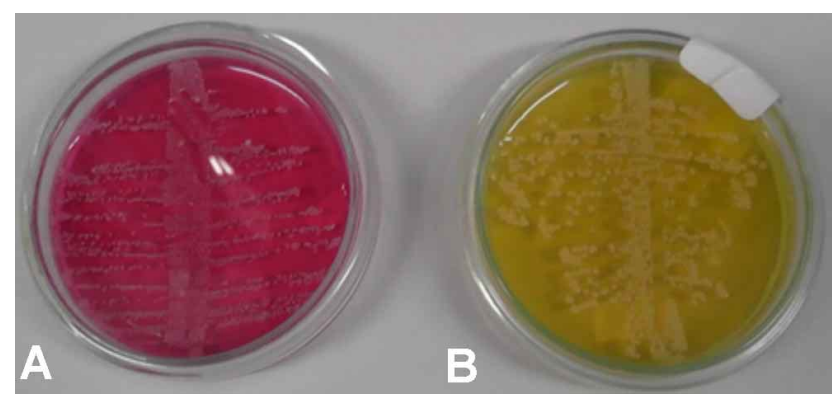

Fig. 1. Crecimiento de colonias del género Staphylococcus aureus en Agar manitol salado. A: Manitol negativo, B: Manitol positivo.

Pruebas de confirmación. Después se realizó la prueba coagulasa para confirmar la identificación de Staphylococcus aureus obtenido de las colonias manitol positivas. Se colocó $1,5 \mathrm{~mL}$ de plasma citratado en un tubo, y se procedió a inocular 3 colonias manitol positivo. Después de 2 a 4 horas de incubación a $37^{\circ} \mathrm{C}$, se observó la presencia o ausencia de coágulos. La formación del coágulo nos indicó positivo a Staphylococcus aureus (Fig. 2).

Antibiograma. Se determinó la sensibilidad antimicrobiana de las cepas aisladas de Staphylococcus aureus. Estas fueron analizadas con el método de difusión en disco de Kirby - Bauer. Los antibióticos empleados fueron: penicilina, oxacilina, amoxilina/ácido clavulánico, ampicilina/sulbactam, vancomicina, ciprofloxacino, gentamicina, eritromicina y cotrimoxazol (BIOLABTEST, Laboratorio Jamper, Perú). Después de 24 horas de incubación a $37^{\circ} \mathrm{C}$ se midieron los halos de inhibición (Fig. 3). Con los resultados obtenidos se confeccionó tablas de distribución de frecuencias y en base a ellas, se realizó el análisis descriptivo.

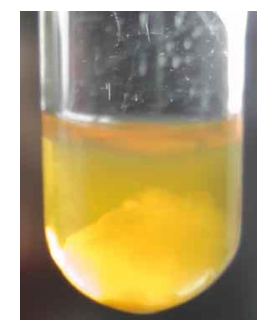

Fig. 2. Prueba de confirmación para la identificación de Staphylococcus aureus: Prueba de la Coagulasa.
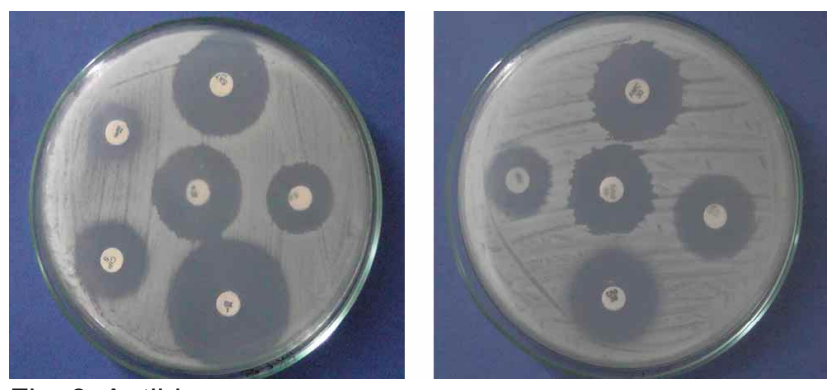

Fig. 3. Antibiograma. 


\section{RESULTADOS}

Se incluyó 64 participantes seleccionados por muestreo aleatorio, $38(59,4 \%)$ pertenecían al sexo femenino y $26(40,6 \%)$ al masculino. De las 64 muestras, $32(50 \%)$ de mano, $10(15,6 \%)$ de fosas nasales y $5(7,8 \%)$ de mucosa oral resultaron positivas a Staphylococcus aureus, es decir, fueron Gram positivas, manitol positivo y coagulasa positiva (Fig. 4). Estas muestras de Staphylococcus aureus fueron sometidas al estudio de sensibilidad antimicrobiana.

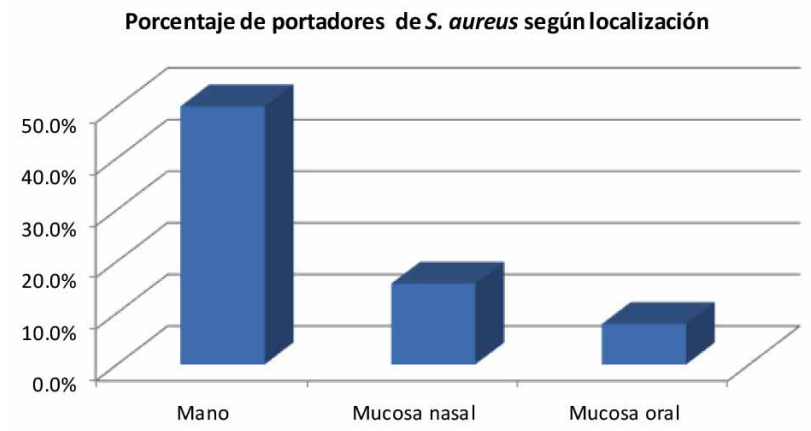

Fig. 4. Porcentaje de portadores de colonias de Staphylococcus aureus aisladas de mano, mucosa nasal y mucosa oral.
En cuanto a las muestras provenientes de mano, el estudio de sensibilidad antimicrobiana indicó un patrón de resistencia al 90,6\% a penicilina, $6,3 \%$ a oxacilina, $3,1 \%$ a amoxicilina/ácido clavulánico, 3,1\% a ampicilina/sulbactam, 15,6 \% a vancomicina, $0,0 \%$ a ciprofloxacino, $6,3 \%$ a gentamicina, $0,0 \%$ a sulfametoxazol, $31,3 \%$ a cotrimoxazol y $34,4 \%$ a eritromicina (Tabla I).

Con respecto a las muestras provenientes de mucosa nasal, el estudio de sensibilidad antimicrobiana indicó un patrón de resistencia al $90,0 \%$ a penicilina, $0,0 \%$ a oxacilina, $10,0 \%$ a amoxicilina/ácido clavulánico, 10,0\% a ampicilina/ sulbactam, $10,0 \%$ a vancomicina, $0,0 \%$ a ciprofloxacino, $0,0 \%$ a gentamicina, $0,0 \%$ a sulfametoxazol, $10,0 \%$ a cotrimoxazol y $70,0 \%$ a eritromicina (Tabla II).

Las muestras provenientes de mucosa oral mostraron un patrón de resistencia al $100,0 \%$ a penicilina, $20,0 \%$ a oxacilina, $0,0 \%$ a amoxicilina/ácido clavulánico, 20,0\% a ampicilina/sulbactam, 20,0\% a vancomicina, $0,0 \%$ a ciprofloxacino, $0,0 \%$ a gentamicina, $0,0 \%$ a sulfametoxazol, $0,0 \%$ a cotrimoxazol y $20,0 \%$ a eritromicina (Tabla III).

Tabla I. Análisis de sensibilidad antimicrobiana en cepas de Staphylococcus aureus aisladas de mano. N: Frecuencia

\begin{tabular}{|c|c|c|c|c|c|c|}
\hline \multirow[b]{2}{*}{ Antimicrobianos } & \multicolumn{2}{|c|}{ Sensibilidad } & \multicolumn{2}{|c|}{ Intermedia } & \multicolumn{2}{|c|}{ Resistencia } \\
\hline & $\mathrm{N}$ & $\%$ & $\mathrm{~N}$ & $\%$ & $\mathrm{~N}$ & $\%$ \\
\hline Penicilina & 3 & 9,4 & 0 & 0,0 & 29 & 90,6 \\
\hline Oxacilina & 27 & 84,4 & 3 & 9,4 & 2 & 6,3 \\
\hline Amoxicilina/ácido clavulánico & 27 & 84,4 & 4 & 12,5 & 1 & 3,1 \\
\hline Ampicilina/sulbactam & 26 & 81,3 & 5 & 15,6 & 1 & 3,1 \\
\hline Vancomicina & 27 & 84,4 & 0 & 0,0 & 5 & 15,6 \\
\hline Ciprofloxacino & 31 & 96,9 & 1 & 3,1 & 0 & 0,0 \\
\hline Gentamicina & 27 & 84,4 & 3 & 9,4 & 2 & 6,3 \\
\hline Sulfametoxazol & 32 & 100,0 & 0 & 0,0 & 0 & 0,0 \\
\hline Cotrimoxazol & 20 & 62,5 & 2 & 6,3 & 10 & 31,3 \\
\hline Eritromicina & 7 & 21,9 & 14 & 43,8 & 11 & 34,4 \\
\hline
\end{tabular}

Tabla II. Análisis de sensibilidad antimicrobiana en cepas de Staphylococcus aureus aisladas de mucosa nasal. N: Frecuencia

\begin{tabular}{|c|c|c|c|c|c|c|}
\hline \multirow[b]{2}{*}{ Antimicrobianos } & \multicolumn{2}{|c|}{ Sensibilidad } & \multicolumn{2}{|c|}{ Intermedia } & \multicolumn{2}{|c|}{ Resistencia } \\
\hline & $\mathrm{N}$ & $\%$ & $\mathrm{~N}$ & $\%$ & $\mathrm{~N}$ & $\%$ \\
\hline Penicilina & 1 & 10,0 & 0 & 0,0 & 9 & 90,0 \\
\hline Oxacilina & 10 & 100,0 & 0 & 0,0 & 0 & 0,0 \\
\hline Amoxicilina/ácido clavulánico & 9 & 90,0 & 0 & 0,0 & 1 & 10,0 \\
\hline Ampicilina/sulbactam & 8 & 80,0 & 1 & 10,0 & 1 & 10,0 \\
\hline Vancomicina & 9 & 90,0 & 0 & 0,0 & 1 & 10,0 \\
\hline Ciprofloxacino & 10 & 100,0 & 0 & 0,0 & 0 & 0,0 \\
\hline Gentamicina & 10 & 100,0 & 0 & 0,0 & 0 & 0,0 \\
\hline Sulfametoxazol & 10 & 100,0 & 0 & 0,0 & 0 & 0,0 \\
\hline Cotrimoxazol & 8 & 80,0 & 1 & 10,0 & 1 & 10,0 \\
\hline Eritromicina & 2 & 20,0 & 1 & 10,0 & 7 & 70,0 \\
\hline
\end{tabular}


MÁRQUEZ-OVIEDO, A.; ROMERO-COASACA, A. C.; REQUENA-MENDIZÁBAL, M. F.; MARTÍNEZ-CADILLO, E.; MONGRUT-VILLEGAS, U.; AYÓN-HARO, E. R. \& GUARDIAHUAMANÍ, A. S. Resistencia de cepas de Staphylococcus aureus aisladas de la mucosa oral de una población joven peruana. Int. J. Odontostomat., $15(3): 634-638,2021$.

Tabla III. Análisis de sensibilidad antimicrobiana en cepas de Staphylococcus aureus aisladas de mucosa oral. N: Frecuencia

\begin{tabular}{lcccccc}
\hline Antimicrobianos & $\mathrm{N}$ & $\begin{array}{c}\text { Sensibilidad } \\
\%\end{array}$ & $\mathrm{~N}$ & $\begin{array}{c}\text { Intermedia } \\
\%\end{array}$ & $\mathrm{~N}$ & $\begin{array}{c}\text { Resistencia } \\
\%\end{array}$ \\
\hline Penicilina & 0 & 0,0 & 0 & 0,0 & 5 & 100,0 \\
Oxacilina & 4 & 80,0 & 0 & 0,0 & 1 & 20,0 \\
Amoxicilina/ácido clavulánico & 3 & 60,0 & 2 & 40,0 & 0 & 0,0 \\
Ampicilina/sulbactam & 3 & 60,0 & 1 & 20,0 & 1 & 20,0 \\
Vancomicina & 4 & 80,0 & 0 & 0,0 & 1 & 20,0 \\
Ciprofloxacino & 5 & 100,0 & 0 & 0,0 & 0 & 0,0 \\
Gentamicina & 5 & 100,0 & 0 & 0,0 & 0 & 0,0 \\
Sulfametoxazol & 5 & 100,0 & 0 & 0,0 & 0 & 0,0 \\
Cotrimoxazol & 5 & 100,0 & 0 & 0,0 & 0 & 0,0 \\
Eritromicina & 2 & 40,0 & 1 & 20,0 & 1 & 20,0 \\
\hline
\end{tabular}

\section{DISCUSIÓN}

Este trabajo tuvo como finalidad determinar los datos sobre sensibilidad y resistencia de Staphylococcus aureus frente a diferentes antimicrobianos en portadores asintomáticos jóvenes de nuestro medio. Estos datos son importantes para obtener un mayor control y seguimiento de la emergencia de cepas de Staphylococcus aureus resistentes.

En este estudio se encontró una alta resistencia a la penicilina, resultado que está de acuerdo con estudios anteriores (Zelaya-Trebejo et al.; González et al., 2007) donde encontraron una resistencia en 100 $\%$ y $97,9 \%$, respectivamente. Se ha descrito que la resistencia a las penicilinas es debido a la producción de la $\beta$-lactamasa bajo el control de un plásmido. El plásmido se transfiere por transducción y quizá también por conjugación (Brooks et al., 2008). La alta resistencia a la penicilina obtenida en el presente estudio se debió probablemente a la presencia de $\beta$ lactamasa en estas cepas.

La resistencia escasa a oxacilina en el presente trabajo se podría atribuir a la ausencia del gen mecA que codifica una proteína de unión a la penicilina de baja afinidad a los antibióticos $\beta$-lactámicos (Brooks et al.). Estos resultados difieren del reporte de Luján (2003) que encontró $100 \%$ de resistencia a oxacilina en pacientes hospitalizados. Estas diferencias se deben principalmente a los tratamientos prolongados de antibióticos recibidos en estos pacientes que trae como consecuencia una mayor resistencia a oxacilina.

También se halló una resistencia a la gentamicina de $6,3 \%$ de las muestras provenientes de mano. Resultados similares se presentaron en muestras de fo- sas nasales en niños (2,8 \%). Por otra parte, Callisaya et al. (2007) encontraron porcentajes mayores (11,2\%) en pacientes de un hospital. La antibioticoterapia prolongada en estos pacientes puede aumentar el porcentaje de resistencia a este antibiótico. Se ha reportado que la resistencia se debe a que la gentamicina es modificada por medio de acetilación, adenilación o fosforilación y esto interfiere en su unión a los ribosomas impidiendo la biosíntesis normal de proteínas de Staphylococcus aureus (Ubukata et al. 1984).

Un hallazgo interesante fue la presencia de resistencia a la vancomicina en un $15,6 \%, 10 \%$ y $20 \%$ de las muestras provenientes de mano, mucosa nasal y mucosa oral, respectivamente. Estos resultados contrastan lo reportado por González et al. quienes hallaron $0 \%$ de resistencia a la vancomicina. Similarmente, Mamani et al. (2006) y Otth et al. (2008) encontraron un $0 \%$ de resistencia en pacientes hospitalizados y ambulatorios. La aparición de cepas resistentes a la vancomicina es una preocupación debido a una potencial infección no tratable. Se sugiere realizar más estudios para identificar el gen de resistencia a vancomicina vanA y el gen de nafcilina mecA para confirmar si se trata de una cepa de Staphylococcus aureus resistente a la vancomicina (Loomba et al.).

Es recomendable realizar estudios obteniendo muestras de las manos e hisopado faríngeo de pacientes que acuden a una cita odontológica. Los datos de sensibilidad y resistencia antimicrobiana contribuirían a un eficaz tratamiento de infecciones odontógenas. Este trabajo contribuye con una información en jóvenes que sirve para controlar la aparición de cepas resistentes a la vancomicina. 
MÁRQUEZ-OVIEDO, A.; ROMERO-COASACA, A. C.; REQUENA-MENDIZÁBAL, M. F.; MARTíNEZ-CADILLO, E.; MONGRUT-VILLEGAS, U.; AYÓN-HARO, E. R. \& GUARDIAHUAMANÍ, A. S. Resistencia de cepas de Staphylococcus aureus aisladas de la mucosa oral de una población joven peruana. Int. J. Odontostomat., 15(3):634-638, 2021.

Se concluye que las muestras provenientes de las manos presentaron la mayor prevalencia de Staphylococcus aureus en comparación a las muestras de mucosa oral y nasal en individuos jóvenes asintomáticos. Se encontró una alta prevalencia en la sensibilidad a amoxicilina/ácido clavulánico, gentamicina, co-trimoxazol, oxacilina, vancomicina y ampicilina/sulbactam de las tres muestras provenientes de los portadores asintomáticos. Este estudio reveló que el porcentaje de resistencia a vancomicina es más alto en comparación a otros estudios reportados anteriormente.

AGRADECIMIENTOS. A la Sra. Katerina Alhuay Zevallos por su contribución en el servicio técnico.

MÁRQUEZ-OVIEDO, A.; ROMERO-COASACA, A. C.; REQUENA-MENDIZÁBAL, M. F.; MARTÍNEZ-CADILLO, E.; MONGRUT-VILLEGAS, U.; AYÓN-HARO, E. R. \& GUARDIA-HUAMANÍ, A. S. Resistance of Staphylococcus aureus strains isolated from the oral mucosa in a young Peruvian population. Int. J. Odontostomat., 15(3):634-638, 2021.

ABSTRACT: Staphylococcus aureus is an important pathogen responsible for a variety of infectious diseases. The increase of resistant strains is an issue of concern. There are very few studies reporting the resistant strains isolated from oral cavity. Aim: To determine the antimicrobial susceptibility of Staphylococcus aureus isolated from oral mucosa of Peruvian young healthy carriers. The study included 64 healthy individuals whose oral mucosa, nasal mucosa and hand swabs were collected and seeded in salt mannitol agar for primary isolation. Then, coagulase test was performed to confirm the presence of Staphylococcus aureus. After that, colonies of Staphylococcus aureus underwent antibiogram by using the Kirby Bauer method. The prevalence of Staphylococcus aureus positive strains isolated from oral mucosa, nasal mucosa, and hand, was $7.8 \%, 15.6 \%, 50.0 \%$, respectively. There was less than $40 \%$ and $11 \%$ of prevalence of susceptibility to erythromycin and penicillin, respectively, in the three studied samples. There was equal or more than $60 \%$ of prevalence of susceptibility to amoxicillin/clavulanic acid, cotrimoxazole, gentamicin, oxacillin, ciprofloxacin, vancomycin and ampicillin/sulbactam in all the studied samples. Vancomycin resistance was found in $10-20 \%$ of all samples. A high prevalence in susceptibility to amoxicillin/ clavulanic acid, gentamicin, co-trimoxazole, oxacillin, ciprofloxacin, vancomycin and ampicillin /sulbactam in healthy carriers was found. Vancomycin resistance percentage is higher than previous reports.

KEY WORDS: Staphylococcus aureus, microbial sensitivity tests, young adult.

\section{REFERENCIAS BIBLIOGRÁFICAS}

Brooks, G.; Carroll, K.; Butel, J. \& Morse, S. Microbiología médica de Jawetz, Melnick y Adelberg. $19^{\mathrm{a}}$ ed. Editorial Manual Moderno, México, 2008.

Callisaya, H.; Sarmiento, Z. \& Choque, H. Prevalencia de portadores nasales de Staphylococcus aureus en el personal de limpieza del Hospital Obrero. Biofarbo, 15(1):55-60, 2007.

González, A. M.; Juárez, G. I.; González, M. L. \& Nadal, B. L. Frecuencia de colonización de Staphylococcus aureus resistente a meticilina en un grupo de niños en edad escolar. Rev. Enfer. Infec. Pediatr., 20(80):86-91, 2007.

Loomba, P. S.; Taneja, J. \& Mishra, B. Methicillin and Vancomycin Resistant S. aureus in Hospitalized Patients. J. Glob. Infect. Dis., 2(3):275-83, 2010.

Luján, D. Evaluación de Staphylococcus aureus multirresistente en pacientes hospitalizados en el Instituto de Enfermedades Neoplásicas. Rev. Per. Enferm. Infecc. Trop., 3(1):10-3, 2003.

Mamani, E.; Lujan, D. \& Pajuelo, G. Perfil de sensibilidad y resistencia de Staphylococcus aureus. Experiencia en el Hospital Nacional Hipólito Unanue. An. Fac. med., 67(2):120-4, 2006.

Mendoza, C. A.; Velásquez, R.; Mercado, D. L.; Ballón, J. \& Maguiña, C. Susceptibilidad antimicrobiana de Staphylococcus aureus sensible, con sensibilidad "BORDERLINE" y resistentes a la meticilina. Rev. Med. Hered., 14(4):181-5, 2003.

Otth, L.; Wilson, M.; Bustamante, N.; Fernández, H. \& Otth, C. Susceptibilidad antimicrobiana y patrones de resistencia de Staphylococcus aureus aislados de pacientes y portadores aislados en la ciudad de Valdivia, Chile. Rev. Chil. Infect., 25(3):1758, 2008.

Ubukata, K.; Yamashita, N.; Gotoh, A. \& Konno, M. Purification and characterization of aminoglycoside-modifying enzymes from Staphylococcus aureus and Staphylococcus epidermidis. Antimicrob. Agents Chemother., 25(2):754-9, 1984.

Zelaya-Trebejo, L.; Zelaya-Vargas, J.; Miranda-Soberón, U.; Guillermo-Albites, J. \& Hernández-Zúñiga D. Portadores intrahospitalarios de Staphylococcus aureus y sensibilidad a los antimicrobianos. Rev. Per. Enferm. Infecc. Trop., 1(1), 2001.

Dirección para correspondencia:

Esperanza Raquel Ayón Haro, DDS, PhD

Laboratorio de Microbiología

Facultad de Odontología

Universidad de San Martín de Porres.

Av. Las Calandrias 151291 - Santa Anita

Lima 43

PERÚ

E-mail: eayonh@usmp.pe 